Research Paper

\title{
Combined Features Based on Preoperative Controlling Nutritional Status Score and Circulating Tumour Cell Status Predict Prognosis for Colorectal Cancer Patients Treated with Curative Resection
}

\author{
Chaogang Yang ${ }^{1,2,3^{*}}$, Chen Wei ${ }^{1,2,3^{*}}$, Shuyi Wang ${ }^{1,2,3^{*}}$, Song Han', Dongdong Shi ${ }^{1,2,3}$, Chunxiao Zhang ${ }^{1,2,3}$, \\ Xiaobin Lin 1,2,3, Rongzhang Dou 1,2,3, Bin Xiong1,2,3 \\ 1. Department of Gastrointestinal Surgery \& Department of Gastric and Colorectal Surgical Oncology, Zhongnan Hospital of Wuhan University, Wuhan 430071, China; \\ 2. Hubei Key Laboratory of Tumour Biological Behaviors, Wuhan 430071, China; \\ 3. Hubei Cancer Clinical Study Center, Wuhan 430071, China; \\ 4. Department of Equipment Research and Development, Wuhan YZY Medical Science \& Technology Co., Ltd., Wuhan 430075, China \\ *These authors contributed equally to this work. \\ $\bowtie$ Corresponding author: Dr. Bin Xiong, MD. Email: binxiong1961@whu.edu.cn; Tel.:+862767813152
}

(c) Ivyspring International Publisher. This is an open access article distributed under the terms of the Creative Commons Attribution (CC BY-NC) license (https://creativecommons.org/licenses/by-nc/4.0/). See http://ivyspring.com/terms for full terms and conditions.

Received: 2019.01.30; Accepted: 2019.03.13; Published: 2019.05.12

\begin{abstract}
Background: The preoperative controlling nutritional status (CONUT) score and circulating tumour cell (CTC) status are associated with poor prognosis of colorectal cancer (CRC). The aim of the present study is to determine whether the combination of CONUT and CTC (CONUT-CTC) could better predict the prognosis of CRC patients treated with curative resection.

Methods: Preoperative CONUT score was retrospectively calculated in $160 \mathrm{CRC}$ patients who underwent curative resection at Zhongnan Hospital of Wuhan University from 2015 to 2017. Preoperative CTC counts were enumerated from $5 \mathrm{ml}$ peripheral vein blood by a CTCBIOPSY® device. According to the preoperative CONUT and CTC status, the patients were divided into three groups: CONUT-CTC (0), CONUT-CTC (1) and CONUT-CTC (2). The relationship between CONUT score and CTC, as well as the associations of CONUT-CTC status with clinicopathological factors and survival, were evaluated.

Results: Preoperatively, the number and positive rate of CTC were positively correlated with the preoperative CONUT score $(\mathrm{P}<0.01)$. An elevated CONUT-CTC score was significantly associated with deeper tumour invasion $(P=0.025)$, lymphatic vessel invasion $(P=0.002)$, venous invasion $(P<0.001)$ and higher PTNM stage $(\mathrm{P}=0.033)$. Kaplan-Meier analysis and log-rank tests revealed significant decreases in recurrence-free survival (RFS) and cancer-specific survival (CSS) among CRC patients with CONUT-CTC score of 0,1 and $2(P<0.001)$. In PTNM stage-stratified analysis, high CONUT-CTC score was significantly associated with the poor $(\mathrm{P}<0.001)$ and CSS $(\mathrm{P}<0.001)$ of patients with stage III disease, but not correlated with the prognosis of patients with stage II disease (RFS: $P=0.077$; CSS: $P<0.090$ ). Further univariate and multivariate analyses showed that CONUT-CTC was an independent factor affecting patients' RFS [hazard ratio $(\mathrm{HR})=2.66,95 \%$ confidence interval $(\mathrm{Cl}): 1.79-3.96, \mathrm{P}<0.001$ ] and CSS $(\mathrm{HR}=3.75,95 \% \mathrm{Cl}: 2.14-6.57, \mathrm{P}<0.001)$. In time-dependent receiver operating characteristics (ROC) analyses, CONUT-CTC score had a higher area under the ROC curve (AUC) for the prediction of RFS and CSS than did preoperative CONUT score or CTC status.

Conclusion: The preoperative CONUT-CTC score is associated with tumour progression and poor prognosis in patients with CRC treated with curative resection, indicating that better information on CRC prognosis could be obtained from combined preoperative host immune-nutritional status and CTC detection.
\end{abstract}

Key words: controlling nutritional status, circulating tumour cell, prognosis, colorectal cancer, curative resection 


\section{Introduction}

The latest epidemiological data report that colorectal cancer (CRC) is now the third most frequently diagnosed cancer, but CRC is the second leading cause of cancer-related death worldwide, with over 1.8 million new cases and 881,000 deaths occurring in 2018[1]. Despite progressive improvements in early diagnosis and multidisciplinary treatment, recurrence and metastasis remain a very common and usually fatal mode of failure after attempted curative treatment[1-3]. The 5-year relative survival rate for CRC patients still remains approximately $65 \%$, yet about $50 \%$ of CRC patients will eventually develop recurrence and metastasis[2]. One of the key reasons is that CRC is a heterogeneous disease, but current prognostic or predictive factors for CRC do not provide optimal risk stratification, thereby affecting the tailored treatment for individual patients[4]. Therefore, it is necessary to establish novel but reliable biomarker panels that can help predict the risk of metastasis and guide individualized treatment.

Circulating tumour cell (CTC) that shed from the primary tumour mass, circulate through the bloodstream and travel to different organs of body are considered to be the precursors of metastasis[5-7]. As a "liquid biopsy" technology, CTC detection is of important value for getting more insights into metastasis-associated progression[7, 8]. Our group and others have contributed a considerable body of evidence indicating that CTC detection in peripheral blood has great clinical potential for identifying potentially metastatic disease earlier, monitoring treatment response and evaluating patients' clinical outcome in CRC[9-12]. In the process of CTC detection, a fact that cannot be ignored is that the number of CTCs in peripheral blood is extremely low, with one CTC in millions of blood cells[13]. It has been estimated that approximately $1 \times 106$ CTCs per gram of tumour tissue are released into the circulation daily[14], but most of them are recognized and cleared by immune cells in the blood microenvironment; ultimately, less than $0.1 \%$ CTCs can reach distant organs to form metastasis lesion[15]. The above perspective has also been confirmed in our previous studies, which showed that the number of CTCs was negatively correlated with lymphocyte count in peripheral blood[16, 17]. Of note, we also found that CTC count had a negative correlation with decreased prognostic nutrition index (PNI)[18], which is an important indicator reflecting the perioperative nutritional conditions of patients with malignant gastrointestinal tract tumours[19]. According to the above findings, we speculated that preoperative host immune-nutritional status is closely related to the number of CTCs in peripheral blood; focusing on this topic could provide us with more valuable clinical information. Recently, controlling nutritional status (CONUT) score, as a newly proposed scoring system of immune-nutritional status, has gradually attracted clinicians' attention[20]. It consists of three blood parameters, including the serum albumin levels, total peripheral lymphocyte counts and total cholesterol concentration, which are representative markers of protein reserves, calorie deficiency, and impaired immune defences, respectively[20]. Emerging evidence has demonstrated that CONUT score was associated with the prognosis of patients with solid tumours[21], including CRC[22-24]. However, the relationship between preoperative CONUT score and CTC remains unknown, and the combined use of these two indices (CONUT-CTC) for evaluating the prognosis of CRC patients treated with curative resection has not yet been explored.

In the present study, we found that, for CRC patients treated with curative resection, the number and positive rate of CTC were positively correlated with the preoperative CONUT score, and an elevated CONUT-CTC score was significantly associated with multiple unfavourable clinicopathological parameters and poor prognosis. Furthermore, we also demonstrated that preoperative CONUT-CTC score could be a better indicator for predicting the recurrence-free survival (RFS) and cancer-specific survival (CSS) of CRC patients. These data indicate that the preoperative CONUT-CTC scoring system is a valuable postoperative prognosticator for CRC patients treated with curative resection and may help identify high-risk patients for rational therapy and timely follow-up.

\section{Materials and Methods}

\section{Study population}

All CRC patients who underwent curative resection at Zhongnan Hospital of Wuhan University from April 2015 to April 2017 were carefully reviewed retrospectively. The eligibility criteria were as follows: (1) histopathologically confirmed CRC; (2) underwent curative resection; (3) with available preoperative CTC detection data; (4) without any neoadjuvant chemotherapy and/or radiotherapy; (5) adequate and available medical record and following-up data. Patients were excluded if they presented with the following: (1) any acute infection or chronic inflammatory disease; (2) any other known autoimmune disorder; (3) receipt of preoperative parenteral nutrition before the blood sample was collected; (4) additional unplanned resection; (5) death within 30 days of surgery.

All data on patient demographics, laboratory, 
pathologic and treatment data were retrospectively obtained from the electronic medical record system. The postoperative pathological tumour-nodemetastasis (TNM) stage of CRC was based on the seventh edition of the American Joint Committee on Cancer (AJCC) cancer staging manual[25]. Postoperative complications and their grade were defined according to the Clavien-Dindo classification[26], which included surgical site infection, anastomotic leakage, any organ disease, any organ infection, abscess, pleural effusion, ascites, bleeding, obstructtion, pancreatic fistula, and lymphorrhea. This study was approved by the Ethical Committee of Zhongnan Hospital of Wuhan University and complied with the standards of the Declaration of Helsinki. Written informed consent was obtained from all patients.

\section{CONUT score and other markers}

Preoperative blood samples were collected and assayed within 2 weeks before surgery. According to the laboratory measurements, including serum albumin, total cholesterol concentrations, and total peripheral lymphocyte counts, preoperative CONUT scores were calculated as in Figure 1A and based on previous reports[20, 23]. The normal cut-offs for serum albumin, total cholesterol, and total lymphocytes were $35 \mathrm{~g} / \mathrm{l}, 180 \mathrm{mg} / \mathrm{dl}$, and $1600 / \mathrm{mm}^{3}$, respectively. Body mass index (BMI) was calculated

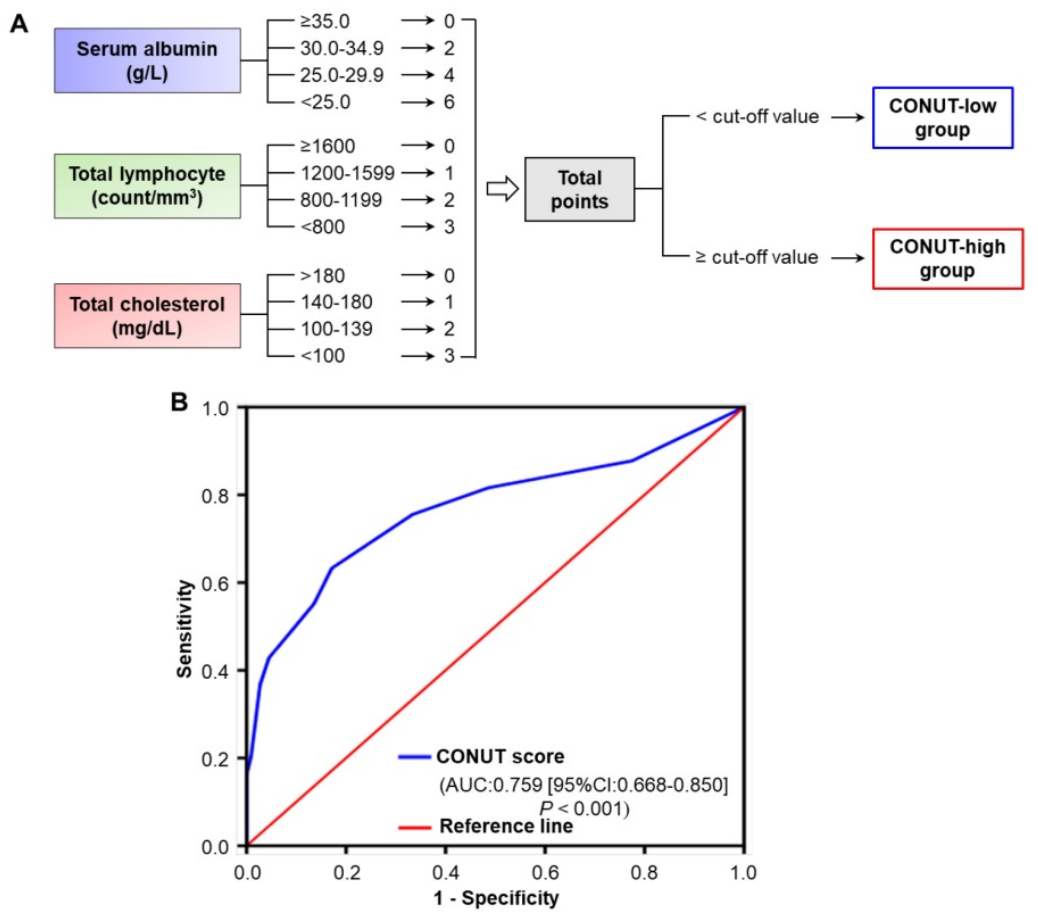

Figure 1. Definition of CONUT score and determination of optimal cut-off value. A. The scoring system for the CONUT scale. According to the cut-off value by ROC analysis, CRC patients were divided into two groups, the CONUT-low group and -high group. B. ROC analysis of the optimal cut-off value of CONUT score based on patients' cancer-specific survival. Abbreviations: CRC, colorectal cancer; CONUT, controlling nutritional status; ROC, receiver operating characteristic; AUC, area under curve; $\mathrm{Cl}$, confidence interval. from preoperative patient height and weight, as measured by our clinical staff on the date of admission. The patients were divided into three BMI groups, as indicated by the World Health Organization: $<18.5 \mathrm{~kg} / \mathrm{m}^{2}, 18.5-24.9 \mathrm{~kg} / \mathrm{m}^{2}$, and $\geq 25 \mathrm{~kg} / \mathrm{m}^{2}$. The cut-off values for serum carcinoembryonic antigen (CEA), carbohydrate antigen (CA) 125 and CA 199 levels were defined as $5.0 \mathrm{ng} / \mathrm{ml}, 35 \mathrm{U} / \mathrm{ml}$ and $37 \mathrm{U} / \mathrm{ml}$, respectively, based on the recommendations of the measuring kit our institute adopted.

\section{CTC isolation and identification}

CTC isolation was performed using the CTCBIOPSY® device (Wuhan YZY Medical Science and Technology Co., Ltd., Wuhan, China), as described in our previous studies[11, 16]. The specific implementation was completed by the professional staff of Wuhan YZY Medical Science and Technology Company according to the manufacturer's instruction. In brief, $5 \mathrm{ml}$ peripheral blood samples from each patient were collected in EDTA-containing tubes (BD, USA) and diluted up to $15 \mathrm{ml}$ with $0.9 \%$ physiological saline containing $0.2 \%$ paraformaldehyde; then, the samples were transferred to tubes with an $8 \mu \mathrm{m}$ diameter aperture membrane. After being filtered by positive pressure from 12 to $20 \mathrm{mmHg}$, the candidate CTCs adhered to the membrane and were identified by three-colour immunofluorescence staining, as described in our previous study[16]. Briefly, membranes with CTC were transferred to glass slides and were fixed with 4\% PFA for 5 minutes. After washing the membrane with $\mathrm{BD}$ wash buffer (BD, USA) three times, $100 \mu \mathrm{l}$ Cytofix/ Permeabilization Kit reagent (BD, USA) was added on the membrane for 20 minutes to allow for intracellular staining. Next, $10 \%$ goat serum was added to block for one hour, followed by discarding the serum and adding either the primary mouse antibody against FITC-CK (1:100; Abcam, USA) and rat antibody against PE-CD45 (1:100; Santa, USA) for incubation overnight at $4^{\circ} \mathrm{C}$. The membrane was washed with $\mathrm{BD}$ wash buffer, followed by the addition of the secondary Alexa Fluor 488-conjugated goat anti-mouse IgG (1:100; Invitrogen, USA) and Alexa Fluor 546-conjugated goat anti-rat IgG (1:200; Invitrogen, USA). The nuclei were stained with Hoechst 33342 (1:500; Sigma, USA) and incubated for one hour; then, the membrane was washed three times with BD wash buffer. 
Finally, CTC were imaged and enumerated using a fluorescence microscopy (IX81; Olympus, Tokyo, Japan). CTCs captured on membranes were photographed using IPP software (Media Cybernetics Inc., Silver Spring, MD, USA). CK+/CD45-/Hoechst+ cells were defined as CTCs, while CK-/CD45+/ Hoechst + cells were regarded as white blood cells (WBC). Based on the results of our previous study, CTC count $\geq 1$ was defined as CTC-positive[16].

\section{Definition of preoperative CONUT-CTC score}

According to the status of preoperative CONUT score and CTC, the CONUT-CTC score was calculated as follows: the patients with CONUT-high (CONUT score $\geq 3$ ) and CTC-positive (CTC count $\geq 1$ ) were assigned a score of 2; the patients with only CONUT-high (CONUT score $\geq 3$ ) or CTC-positive (CTC count $\geq 1$ ) were assigned a score of 1 ; the patients with CONUT-low (CONUT score $<3$ ); and CTC-negative patients $($ CTC count $=0)$ were assigned a score of 0 .

\section{Surveillance and follow-up strategy}

Adjuvant treatments, including chemotherapy (5-fluorouracil-based combination chemotherapy) and radiotherapy, were planned according to the postoperative pathological tumour stage, doctor's selection and patient's desire. Following the recommendations in recognized guidelines[27-29], all patients were recommended for postoperative monitoring by physical examination and laboratory tests, including tests for tumour markers (such as CEA and CA199) every 3 months for the first 2 years, every 6 months for the next 3 years, and annually thereafter. Imaging, most frequently computer tomography (CT) of the thorax, abdomen and pelvis, was recommended for performing at a minimum of every 12 months for at least 3 years. Colonoscopy was recommended for typically performing within the first year after surgery and then repeated every 3 to 5 years unless advanced tumours were identified. If necessary, further evaluations, such as positron emission tomography (PET) or magnetic resonance imaging (MRI), were initiated to better identify recurrence. Follow-up data were available from patient files or by telephone interview with the patients or guardians. All patients were monitored either until September 31, 2018 or their death. RFS was determined from the date of resection to the date of tumour recurrence or distant metastasis. CSS was defined as the time in months between the operation and death caused by GC or last follow-up. Tumour recurrence was definitively diagnosed based on the appearance of new lesions on CT, MRI, and/or PET images and/or histological confirmation through biopsy after reviewing the radiographic reports.

\section{Statistical analysis}

Categorical data were summarized by a number (\%). Continuous variables are presented as median with ranges (minimum, maximum) or mean \pm standard deviation (mean \pm SD). The optimal cut-off value for CONUT score was determined by the maximum of Youden index (sensitivity + specificity-1) based on receiver operating characteristics (ROC) curve analyses. The relationships between the CONUT-CTC score and the clinicopathologic characteristics were analysed with the Pearson $\chi^{2}$ test. Correlation between CONUT score and CTC count was assessed with Spearman's correlation coefficient. The overall cumulative probability of RFS and CSS were calculated using the Kaplan-Meier method, and differences in survival rates among groups with different preoperative CONUT score, CTC status or CONUT-CTC score were determined using the Log-Rank test. The variables associated with RFS and CSS that were significant in univariate analyses were selected for multivariate Cox proportional hazards model to identify independent prognostic factors. ROC analyses were further used to compare the prognostic value of independent prognostic factors, and AUC were calculated. Hazard ratios (HR) estimated from the Cox analysis are reported as relative risks with corresponding 95\% confidence intervals (CIs). A two-sided $\mathrm{P}$ value $<0.05$ was considered statistically significant. All statistical analyses were performed with SPSS software (version 22.0, SPSS Institute, Chicago, IL, USA).

\section{Results}

\section{Patient characteristics}

A total of 160 eligible patients, including 90 $(56.3 \%)$ males and $70(43.7 \%)$ females, were enrolled, with ages ranging from 28 to 81 years (Mean \pm SD: $58.4 \pm 11.8$ years). Among all the included patients, tumours were located in the colon in $93(58.1 \%)$ patients and in the rectum in $67(41.9 \%)$ patients. There were $38(23.8 \%), 55(34.4 \%)$, and 67 (41.8\%) tumours with well, moderate, and poor grades, respectively. T1, T2, T3 and T4 grade tumours were found in $4(2.5 \%), 20(12.5 \%), 62(38.8 \%)$, and 74 $(46.2 \%)$ patients, respectively; stage I, II, and III disease was identified in 13 (8.1\%), 78 (48.8\%), and 69 $(43.1 \%)$ patients, respectively. Based on the illustration in Figure 1A, the preoperative CONUT scores of all included patients were calculated, respectively. Furthermore, according to the optimal cut-off value determined by ROC analysis, 3 was defined as the optimal cut-off value for CONUT score [specificity: 0.821 , sensitivity: 0.625 ; area under the 
curve $(\mathrm{AUC})=0.759,95 \% \mathrm{CI}: 0.668-0.850, \mathrm{P}<0.001]$ (Figure 1B), and $\geq 3$ was defined as a high preoperative CONUT score. There were $86(53.8 \%)$ and $74(46.2 \%)$ patients with low and high preoperative CONUT scores, respectively. Preoperative CTC-negative and -positive status was found in 43 $(26.9 \%)$ and $117(73.1 \%)$ patients, respectively. The CONUT-CTC score was 0 for 34 (21.3\%) patients, 1 for $61(38.1 \%)$ patients, and 2 for $65(40.6 \%)$ patients. The major clinicopathological characteristics of all included patients are summarized in Table S1.

\section{Relationship between preoperative CONUT score and CTC}

Representative images of CTCs (from patient 6 and 83) were shown in Figure 2A. The relationships between preoperative CONUT score and CTC were examined. The results showed a significant positive correlation between serum preoperative CONUT score and CTC count $(\mathrm{r}=0.623, \mathrm{P}<0.001)$ (Figure 2B). In addition, the positive rate of CTC in patients in the preoperative CONUT-high group was significantly higher than that in the CONUT-low group $(\mathrm{P}<0.01)$ (Figure 2C).

\section{Correlation between preoperative CONUT-CTC score and clinicopathological characteristics}

The relationships between preoperative CONUT-CTC score and the clinicopathological characteristics of 160 included CRC patients were presented in Table 1. The data showed that preoperative CONUT-CTC score was significantly correlated with depth of tumour invasion $(\mathrm{P}=0.025)$, $\mathrm{PTNM}$ stage $(\mathrm{P}=0.033)$, lymphatic vessel invasion $(\mathrm{P}=0.002)$ and venous invasion $(\mathrm{P}<0.001)$ but was not correlated with gender, age, BMI, tumour location, tumour size, histopathological type, lymph node metastasis, severe complication, adjuvant chemotherapy, preoperative CEA level, preoperative CA125 level or preoperative CA199 level ( $P>0.05$ for all).
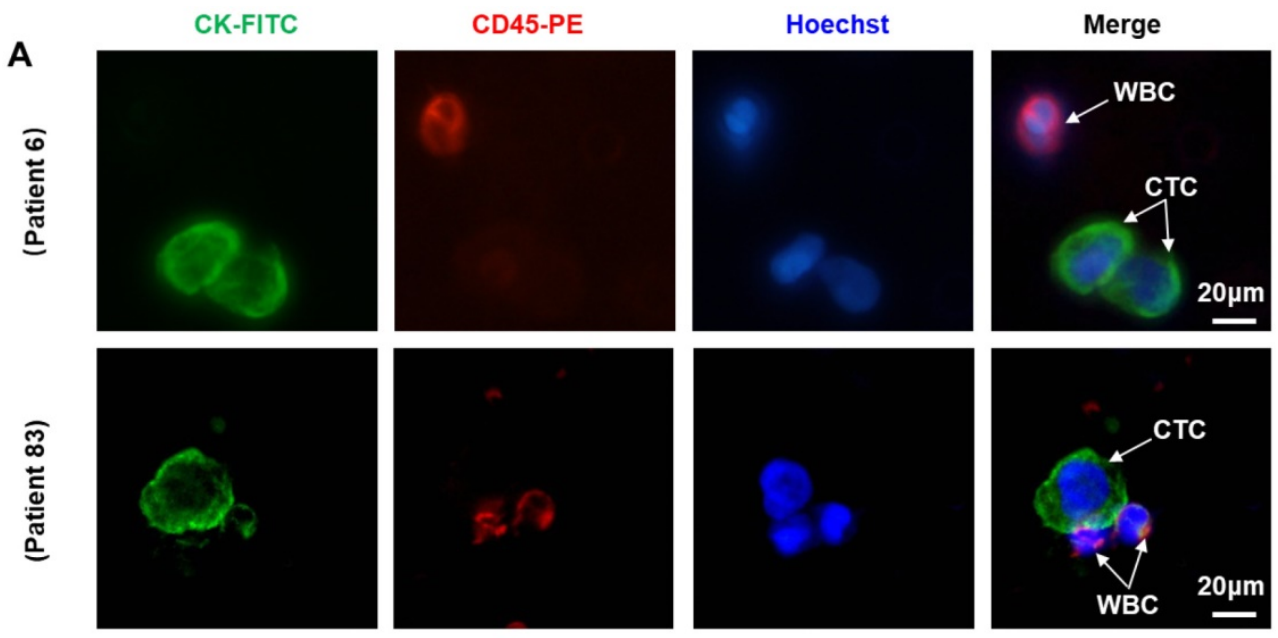

\section{B}
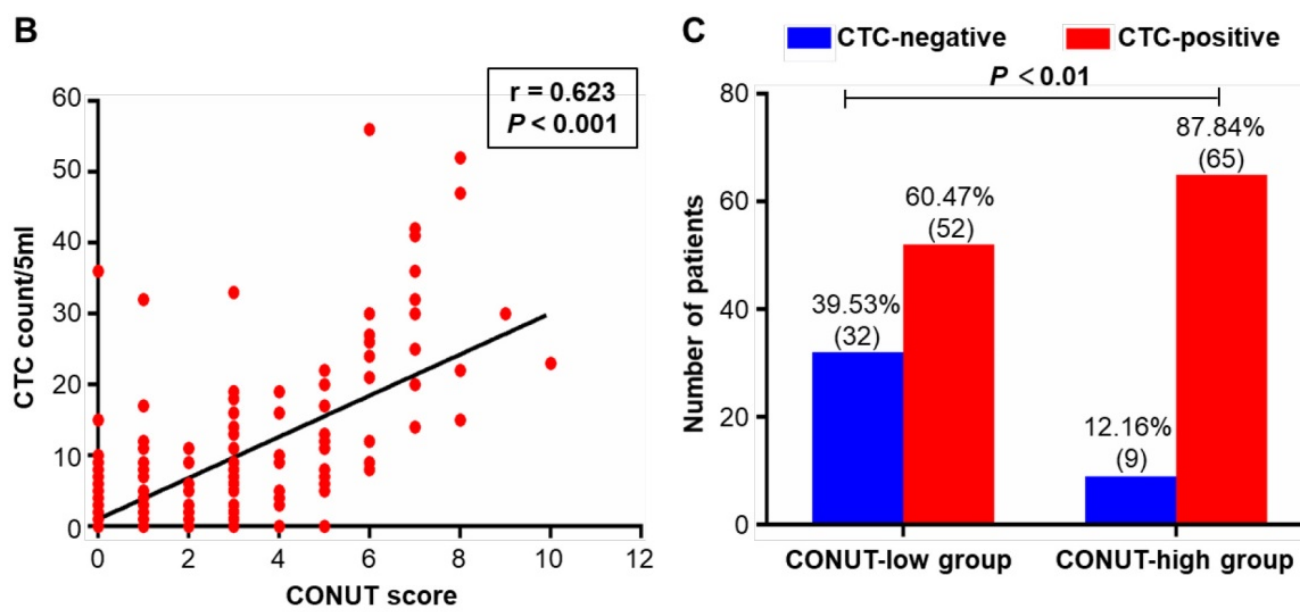

Figure 2. Relationship between preoperative CONUT score and CTC in peripheral blood of CRC patients. A. Representative CTC images from included patients 6 and 83. Three-colour immunofluorescence staining based on FITC-labelled anti-CK, PE-labelled anti-CD45, and Hoechst nuclear staining was applied to identify and enumerate CTC from non-specifically trapped WC. B. Correlation analysis between preoperative CONUT score and CTC count. C. Difference analysis of CTC positive rate between preoperative CONUT-low and -high group. Note: Scale bar: $20 \mu \mathrm{m}$. Abbreviations: CONUT, controlling nutritional status; CTC, circulating tumour cell; CRC, colorectal cancer; FITC, fluorescein isothiocyanate; PE, phycoerythrin; WBC, white blood cell. 
Table 1. Relationships between CONUT-CTC score and clinicopathological characteristics of $160 \mathrm{CRC}$ patients

\begin{tabular}{|c|c|c|c|c|c|}
\hline \multirow[t]{2}{*}{ Characteristics } & \multicolumn{3}{|c|}{ CONUT-CTC score [n (\%)] } & \multirow{2}{*}{$\begin{array}{l}X^{2} \\
\text { value }\end{array}$} & \multirow[t]{2}{*}{ P value } \\
\hline & $\begin{array}{l}\text { Score } 0 \\
(\mathrm{n}=34)\end{array}$ & $\begin{array}{l}\text { Score } 1 \\
(\mathrm{n}=61) \\
\end{array}$ & $\begin{array}{l}\text { Score } 2 \\
(n=65) \\
\end{array}$ & & \\
\hline Gender & & & & 0.195 & 0.907 \\
\hline Male & $18(52.94)$ & $35(57.38)$ & $37(56.92)$ & & \\
\hline Female & $16(47.06)$ & $26(42.62)$ & $28(43.08)$ & & \\
\hline Age (years) & & & & 0.425 & 0.808 \\
\hline$<60$ & $16(47.06)$ & $32(52.46)$ & $35(53.85)$ & & \\
\hline$\geq 60$ & $18(52.94)$ & $29(47.54)$ & $30(46.15)$ & & \\
\hline BMI $\left(\mathbf{k g} / \mathbf{m}^{2}\right)$ & & & & 4.755 & 0.313 \\
\hline$<18.5$ & $15(44.12)$ & $22(36.07)$ & $32(49.23)$ & & \\
\hline$\geq 18.5,<25.0$ & $13(38.24)$ & $33(54.10)$ & $24(36.92)$ & & \\
\hline$\geq 25.0$ & $6(17.64)$ & $6(9.83)$ & $9(13.85)$ & & \\
\hline Tumour location & & & & 3.981 & 0.137 \\
\hline Colon & $16(47.06)$ & $41(67.21)$ & $36(55.38)$ & & \\
\hline Rectal & $18(52.94)$ & $20(32.79)$ & $29(44.62)$ & & \\
\hline Tumour size (cm) & & & & 1.011 & 0.603 \\
\hline$<3$ & $17(50.00)$ & $37(60.66)$ & $37(56.92)$ & & \\
\hline$\geq 3$ & $17(50.00)$ & $24(39.34)$ & $28(43.08)$ & & \\
\hline Histopathological type & & & & 1.114 & 0.573 \\
\hline Well-moderate & $22(64.71)$ & $36(59.02)$ & $35(53.85)$ & & \\
\hline Poor & $12(35.29)$ & $25(40.98)$ & $30(46.15)$ & & \\
\hline Depth of tumour invasion & & & & 7.406 & $0.025^{*}$ \\
\hline T1-T2 & $10(29.41)$ & $8(13.11)$ & $6(9.23)$ & & \\
\hline T3-T4 & $24(70.59)$ & $53(86.89)$ & $59(90.77)$ & & \\
\hline Lymph node metastasis & & & & 5.115 & 0.078 \\
\hline N0-1 & $27(79.41)$ & $41(67.21)$ & $37(56.92)$ & & \\
\hline N2-3 & 7 (20.59) & $20(32.79)$ & $28(43.08)$ & & \\
\hline pTNM stage & & & & 6.831 & $0.033^{*}$ \\
\hline I-II & $23(67.65)$ & $39(63.93)$ & $29(44.62)$ & & \\
\hline III & $11(32.35)$ & $22(36.07)$ & $36(55.38)$ & & \\
\hline Lymphatic vessel invasion & & & & 12.538 & $0.002^{*}$ \\
\hline Negative & $27(79.41)$ & $40(65.58)$ & $29(44.62)$ & & \\
\hline Positive & $7(20.59)$ & $21(34.42)$ & $36(55.38)$ & & \\
\hline Venous invasion & & & & 17.944 & $<0.001^{*}$ \\
\hline Negative & $29(85.29)$ & $37(60.66)$ & $32(49.23)$ & & \\
\hline Positive & $5(14.71)$ & $24(39.34)$ & $33(50.77)$ & & \\
\hline Severe complication & & & & 1.539 & 0.463 \\
\hline Absence & $28(82.35)$ & $50(81.97)$ & $58(89.23)$ & & \\
\hline Presence & $6(17.65)$ & $11(18.03)$ & $7(10.77)$ & & \\
\hline Adjuvant chemotherapy & & & & 4.401 & 0.111 \\
\hline No & $21(61.76)$ & $31(50.82)$ & $26(40.00)$ & & \\
\hline Yes & $13(38.24)$ & $30(49.18)$ & $39(60.00)$ & & \\
\hline Preoperative CEA (ng/ml) & & & & 1.389 & 0.499 \\
\hline$<5$ & $25(73.53)$ & $39(63.93)$ & $47(72.31)$ & & \\
\hline$\geq 5$ & $9(26.47)$ & $22(36.07)$ & $18(27.69)$ & & \\
\hline Preoperative CA125 ( U/ml) & & & & 0.169 & 0.919 \\
\hline$<35$ & $26(76.47)$ & $46(75.41)$ & $51(78.46)$ & & \\
\hline$\geq 35$ & $8(23.53)$ & $15(24.59)$ & $14(21.54)$ & & \\
\hline Preoperative CA199 ( U/ml) & & & & 0.415 & 0.813 \\
\hline$<37$ & $23(67.65)$ & 45 (73.77) & $47(72.31)$ & & \\
\hline$\geq 37$ & $11(32.35)$ & $16(26.23)$ & $18(27.69)$ & & \\
\hline
\end{tabular}

Notes: "indicates $\mathrm{P}<0.05$. CRC, colorectal cancer; CONUT, controlling nutritional status; CTC, circulating tumour cell; BMI, body mass index; TNM, tumour-nodemetastasis; CEA, carcinoembryonic antigen; CA125, carbohydrate antigen 125; CA199; carbohydrate antigen 199.

\section{Survival analysis}

The median follow-up duration was 30 months (range, 6-42 months). By the last follow-up time, 68 $(42.5 \%)$ patients developed disease recurrence, and 47 $(29.3 \%)$ died. The recurrence and death rates of patients in preoperative CONUT-CTC score groups 0 , 1 and 2 were $11.76 \%, 34.43 \%$ and $66.15 \%$ and $2.94 \%$, $19.67 \%$ and $52.31 \%$, respectively, with significant differences between groups $(\mathrm{P}<0.001)$ (Figure 3$)$. The median RFS and CSS were 31.8 (range 30.0-33.5) months and 36.5 (range 35.3-37.8) months, respectively. As expected, traditional factors were associated with CRC patients' RFS and CSS, such as histopathological type, depth of tumour invasion, lymph node metastasis, pTNM stage, lymphatic vessel invasion, venous invasion and adjuvant chemotherapy ( $\mathrm{P}<0.05$ for all) (Table S2 for RFS; Table S3 for CSS). In addition, both high preoperative CONUT score and CTC-positive status were significant correlated with poor RFS (Log-Rank $\mathrm{P}<0.001)$ and CSS (Log-Rank $\mathrm{P}<0.001)$ (Figure S1).

Kaplan-Meier analysis and the Log-Rank test showed that an elevated CONUT-CTC score was significantly associated with shorter RFS (Log-Rank $\mathrm{P}<0.001$ ) (Figure 4A) and CSS (Log-Rank $\mathrm{P}<0.001$ ) (Figure 4B). Stage-specific RFS and CSS are shown in Figure 5. In patients with stage II disease, the mean RFS and CSS time of patients was not significantly different among different preoperative CONUT-CTC score groups, although both of them were shorter in the preoperative CONUT-CTC score of 2 group than that of patients with scores of 1 or 0 (RFS: Log-Rank $\mathrm{P}=0.077$; CSS: Log-Rank $\mathrm{P}<0.090$ ) (Figure 5A and 5B). Among patients with stage III disease, the mean RFS and CSS time of patients with a preoperative CONUT-CTC score of 2 was significantly shorter than that of patients with scores of 1 or 0 (Log-Rank $\mathrm{P}<0.001$ ) (Figure 5C and 5D). However, we did not conduct a subgroup analysis in patients with stage I disease due to the small number of patients.

Univariate and multivariate analyses were further performed to explore the factors associated with RFS and CSS in the included CRC patients. In univariate analyses, traditional clinicopathological features (such as histopathological type, depth of tumour invasion, lymph node metastasis, pTNM stage, lymphatic vessel invasion, venous invasion and adjuvant chemotherapy), as well as preoperative CONUT score, preoperative CTC status and preoperative CONUT-CTC score, were associated with RFS and CSS ( $\mathrm{P}<0.05$ for all) (Table 2). Factors showing significance by univariate analysis were integrated into multivariate Cox proportional hazards analysis. In this study, CONUT score, CTC status and combined features (CONUT-CTC score) were highly correlated. Therefore, two separate multivariate models were generated to avoid the multicollinearity among the above three variables. The results showed that preoperative CONUT score and CTC status were independent prognostic factors for RFS and CSS after excluding other confounding factors $(\mathrm{P}<0.05$ for all). Moreover, the combined features (CONUT-CTC score) were also an independent prognostic factor for 
RFS and CSS in the included patients (RFS: HR=2.66, 95\%CI: 1.79-3.96, P<0.001; CSS: HR=3.75, 95\% CI: 2.14-6.57, $\mathrm{P}<0.001$ ) (Table 3).

\section{Comparison of the predictive value of independent prognostic factors}

ROC analysis was implemented to further evaluate the prognostic performance of the four independent factors in this study. The results showed that preoperative CONUT-CTC score would be better at predicting the RFS and OS of CRC patients compared to preoperative CONUT score, preoperative CTC status and pTNM stage (RFS: $A U C=0.734$, 95\%CI: 0.656-0.812, $\mathrm{P}<0.001$, Figure 6A; CSS: AUC $=0.757,95 \%$ CI: 0.680-0.834, P<0.001, Figure 6B).

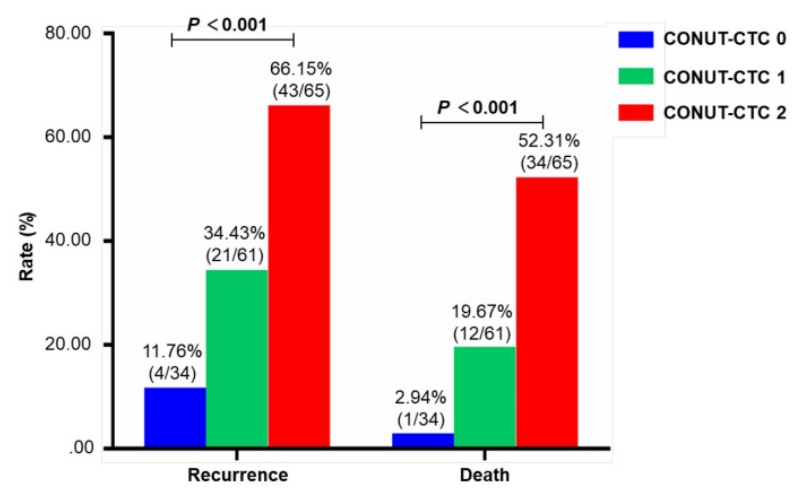

Figure 3. Comparison of the difference of recurrence rate and mortality in different preoperative CONUT-CTC score groups. Abbreviations: CONUT, controlling nutritional status; CTC, circulating tumour cell.
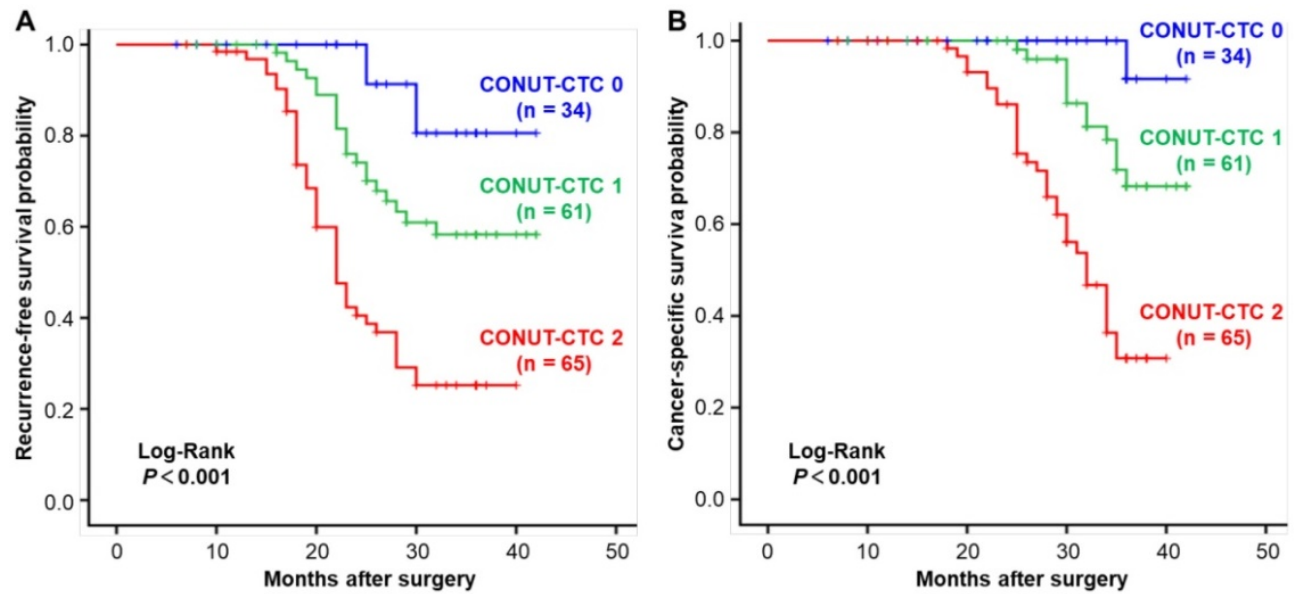

Figure 4. Kaplan-Meier curves for CRC patients in the different preoperative CONUT-CTC score groups. A. Recurrence-free survival. B. Cancer-specific survival. Abbreviations: CRC, colorectal cancer; CONUT, controlling nutritional status; CTC, circulating tumour cell.

Table 2. Univariate analyses of factors associated with recurrence-free survival and cancer-specific survival of CRC patients

\begin{tabular}{|c|c|c|c|c|c|c|}
\hline \multirow[t]{2}{*}{ Factors } & \multicolumn{3}{|c|}{ Recurrence-free survival } & \multicolumn{3}{|c|}{ Cancer-specific survival } \\
\hline & HR & $95 \% \mathrm{CI}$ & P value & HR & $95 \% \mathrm{CI}$ & P value \\
\hline Gender (Male vs. Female) & 0.88 & $0.55-1.43$ & 0.618 & 0.88 & $0.49-1.59$ & 0.680 \\
\hline Age (<60 vs. $\geq 60$ years) & 0.70 & $0.43-1.14$ & 0.151 & 0.85 & $0.48-1.51$ & 0.573 \\
\hline BMI ( $<18.5$ vs. $\geq 18.5,<25.0$ vs. $\left.\geq 25.0 \mathrm{~kg} / \mathrm{m}^{2}\right)$ & 0.88 & $0.62-1.25$ & 0.478 & 0.74 & $0.48-1.15$ & 0.180 \\
\hline Tumour location (Colon vs. Rectal) & 1.48 & $0.86-2.22$ & 0.187 & 1.44 & $0.81-2.54$ & 0.216 \\
\hline Tumour size (<5 vs. $\geq 5 \mathrm{~cm})$ & 1.10 & $0.68-1.78$ & 0.687 & 1.37 & $0.77-2.42$ & 0.287 \\
\hline Histopathological type (Well-moderate vs. Poor) & 1.41 & $1.04-1.93$ & $0.028^{*}$ & 1.47 & $1.01-2.14$ & $0.046^{*}$ \\
\hline Depth of tumour invasion (T1-T2 vs. T3-T4) & 1.56 & $1.07-2.28$ & $0.022^{*}$ & 1.60 & $1.01-2.54$ & $0.048^{*}$ \\
\hline Lymph node metastasis (N0-1 vs. N2-3) & 1.63 & $1.30-2.06$ & $<0.001^{*}$ & 1.62 & $1.22-2.16$ & $0.001^{*}$ \\
\hline pTNM stage (I-II vs. III) & 3.07 & $1.92-4.92$ & $<0.001^{*}$ & 4.08 & $2.19-7.57$ & $<0.001^{*}$ \\
\hline Lymphatic vessel invasion (Negative vs. Positive) & 2.18 & $1.35-3.52$ & $0.002^{*}$ & 2.91 & $1.61-5.29$ & $<0.001^{*}$ \\
\hline Venous invasion (Negative vs. Positive) & 1.94 & $1.20-3.12$ & $0.007^{*}$ & 2.07 & $1.17-3.70$ & $0.013^{*}$ \\
\hline Severe complication (Absence vs. Presence) & 1.34 & $0.72-2.41$ & 0.334 & 1.17 & $0.56-2.41$ & 0.680 \\
\hline Adjuvant chemotherapy (no vs. yes) & 1.87 & $1.14-3.08$ & $0.014^{*}$ & 2.74 & $1.42-5.28$ & $0.003^{*}$ \\
\hline Preoperative CEA ( $<5$ vs. $\geq 5 \mathrm{ng} / \mathrm{ml}$ ) & 1.50 & $0.92-2.46$ & 0.108 & 1.16 & $0.63-2.15$ & 0.631 \\
\hline Preoperative CA125 (<35 vs. $\geq 35 \mathrm{U} / \mathrm{ml})$ & 1.51 & $0.90-2.54$ & 0.116 & 1.62 & $0.89-2.97$ & 0.116 \\
\hline Preoperative CA199 (<37 vs. $\geq 37$ U/ml) ) & 1.45 & $0.89-2.38$ & 0.138 & 1.49 & $0.82-2.68$ & 0.188 \\
\hline Preoperative CONU score (Low vs. High) & 2.79 & $1.69-4.59$ & $<0.001^{*}$ & 4.55 & $2.35-8.81$ & $<0.001^{*}$ \\
\hline Preoperative CTC staus (Negative vs. Positive) & 5.81 & 2.34-14.47 & $<0.001^{*}$ & 8.14 & $1.97-33.57$ & $0.004^{*}$ \\
\hline Preoperative CONUT-CTC score (0 vs. 1 vs. 2$)$ & 2.78 & $1.88-4.13$ & $<0.001^{*}$ & 3.96 & $2.28-6.90$ & $<0.001^{*}$ \\
\hline
\end{tabular}

Notes: "indicates P<0.05. CRC, colorectal cancer; HR, hazard ratio; CI, confidence interval; BMI, body mass index; TNM, tumour-node-metastasis; CEA, carcinoembryonic antigen; CA125, carbohydrate antigen 125; CA199; carbohydrate antigen 199; CONUT, controlling nutritional status; CTC, circulating tumour cell. 
Table 3. Multivariate analyses of factors associated with recurrence-free survival and cancer-specific survival of CRC patients

\begin{tabular}{|c|c|c|c|c|c|c|}
\hline \multirow[t]{2}{*}{ Factors } & \multicolumn{3}{|c|}{ Recurrence-free survival } & \multicolumn{3}{|c|}{ Cancer-specific survival } \\
\hline & HR & $95 \% \mathrm{CI}$ & P value & HR & $95 \% \mathrm{CI}$ & P value \\
\hline \multicolumn{7}{|l|}{ Model 1} \\
\hline Histopathological type (Well-moderate vs. Poor) & 1.33 & $0.97-1.81$ & 0.074 & 1.23 & $0.83-1.83$ & 0.298 \\
\hline Depth of tumour invasion (T1-T2 vs. T3-T4) & 1.18 & $0.72-1.93$ & 0.512 & 0.81 & $0.42-1.55$ & 0.526 \\
\hline Lymph node metastasis (N0-1 vs. N2-3) & 1.14 & $0.76-1.71$ & 0.529 & 0.84 & $0.52-1.37$ & 0.495 \\
\hline pTNM stage (I-II vs. III) & 2.78 & $1.01-7.72$ & $0.049^{*}$ & 4.94 & $1.46-16.69$ & $0.010^{*}$ \\
\hline Lymphatic vessel invasion (Negative vs. Positive) & 1.34 & $0.71-2.54$ & 0.365 & 1.46 & $0.64-3.33$ & 0.371 \\
\hline Venous invasion (Negative vs. Positive) & 0.58 & $0.31-1.07$ & 0.081 & 0.54 & $0.25-1.14$ & 0.107 \\
\hline Adjuvant chemotherapy (No vs. Yes) & 0.74 & $0.32-1.70$ & 0.484 & 1.29 & $0.47-3.57$ & 0.619 \\
\hline Preoperative CONUT score (Low vs. High) & 2.02 & $1.19-3.43$ & $0.010^{*}$ & 3.45 & $1.68-7.10$ & $0.001^{*}$ \\
\hline Preoperative CTC status (Negative vs. Positive) & 4.79 & $1.82-12.60$ & $0.002^{*}$ & 4.70 & $1.05-21.03$ & $0.043^{*}$ \\
\hline \multicolumn{7}{|l|}{ Model 2} \\
\hline Histopathological type (Well-moderate vs. Poor) & 1.27 & $0.93-1.73$ & 0.127 & 1.22 & $0.82-1.80$ & 0.323 \\
\hline Depth of tumour invasion (T1-T2 vs. T3-T4) & 1.12 & $0.69-1.82$ & 0.647 & 0.80 & $0.42-1.53$ & 0.500 \\
\hline Lymph node metastasis (N0-1 vs. N2-3) & 1.12 & $0.75-1.67$ & 0.592 & 0.84 & $0.52-1.37$ & 0.491 \\
\hline pTNM stage (I-II vs. III) & 2.99 & $1.09-8.16$ & $0.033^{*}$ & 5.01 & $1.49-16.82$ & $0.009^{*}$ \\
\hline Lymphatic vessel invasion (Negative vs. Positive) & 1.28 & $0.68-2.39$ & 0.442 & 1.43 & $0.63-3.24$ & 0.390 \\
\hline Venous invasion (Negative vs. Positive) & 0.63 & $0.34-1.16$ & 0.138 & 0.55 & $0.26-1.16$ & 0.117 \\
\hline Adjuvant chemotherapy (No vs. Yes) & 0.77 & $0.34-1.73$ & 0.526 & 1.31 & $0.48-3.60$ & 0.596 \\
\hline Preoperative CONUT-CTC score (0 vs. 1 vs. 2$)$ & 2.66 & $1.79-3.96$ & $<0.001^{*}$ & 3.75 & $2.14-6.57$ & $<0.001^{*}$ \\
\hline
\end{tabular}

Notes: "indicates $\mathrm{P}<0.05$. CRC, colorectal cancer; HR, hazard ratio; CI, confidence interval; TNM, tumour-node-metastasis; CEA, carcinoembryonic antigen; CONUT, controlling nutritional status; CTC, circulating tumour cell.

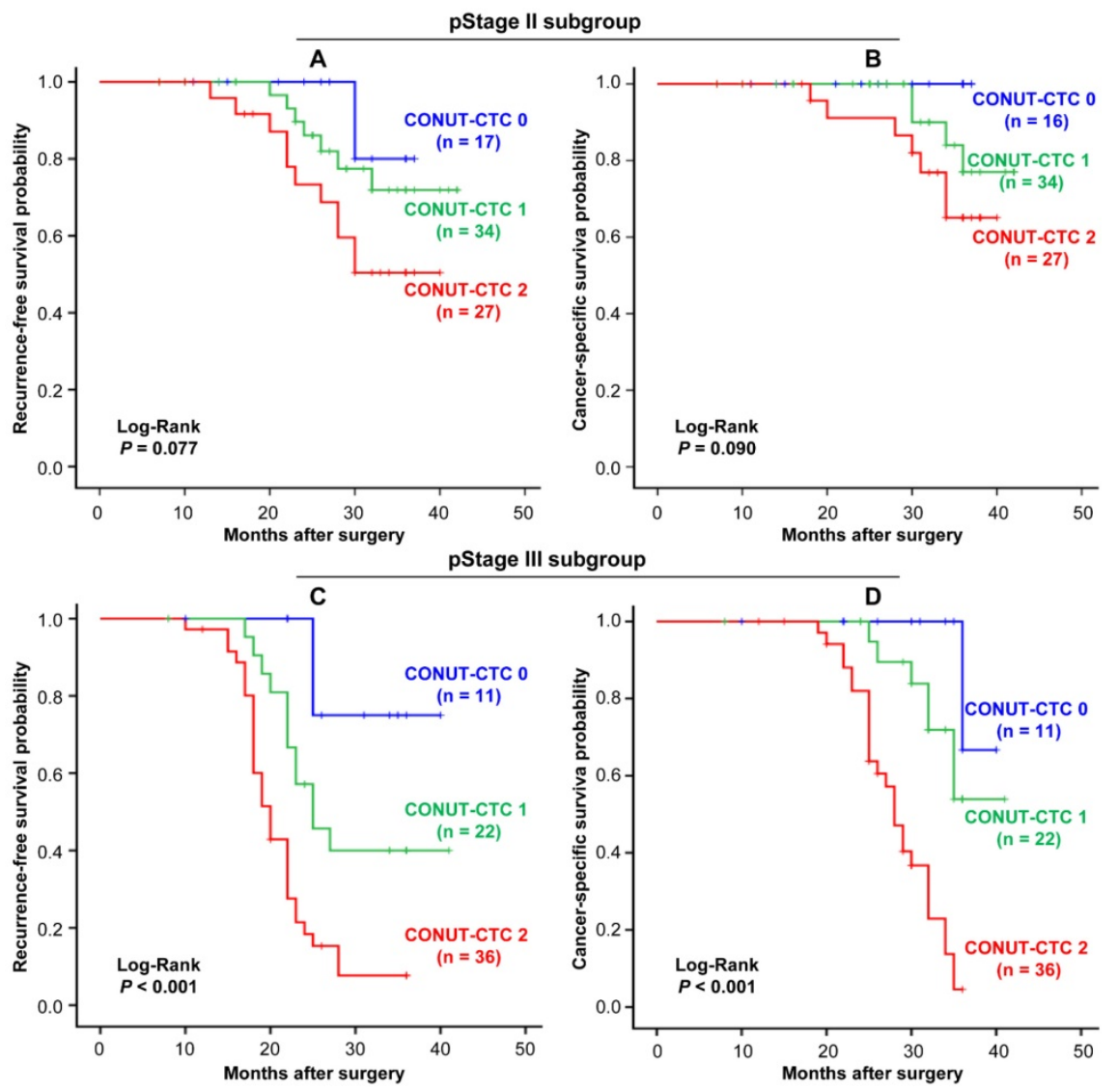

Figure 5. Stage-specific Kaplan-Meier curves for CRC patients in different preoperative CONUT-CTC score groups. A. Recurrence-free survival for pStage I/II. B. Cancer-specific survival for pStage I/II. C. Recurrence-free survival for pStage III. D. Cancer-specific survival for pStage III. Abbreviations: CRC, colorectal cancer; CONUT, controlling nutritional status; CTC, circulating tumour cell.

\section{Discussion}

Despite great progress in understanding the biological behaviour of $\mathrm{CRC}[28,29]$, routine prognostic risk assessment of patients with CRC still relies on the traditional model. Currently, TNM classification is still the most commonly used prognostic predictive tool for CRC patients[25, 30]; 
however, this marker is lacking in regard to reflecting the characteristics of the tumour, which is difficult to generalize due to the individual differences between patients, such as the immune-nutritional status and micrometastatic condition in the blood. Therefore, these traditional prognostic factors have yet to be improved by the incorporation of novel biomarkers, which might be beneficial for individualized treatment of CRC patients. In the present study, we investigated the prognostic value of preoperative CONUT-CTC score, a novel incorporated prognostic system, for 160 CRC patients undergoing curative resection. To the best of our knowledge, this is the first study to uncover that preoperative CONUT-CTC score is associated with tumour progression and can be considered an independent marker for better predicting the prognosis of patients with CRC treated with curative resection.

The CONUT score is proposed as a blood-based marker for reflecting host immune-nutritional status[20,23], and growing evidence has shown that higher preoperative CONUT score indicates worse nutritional condition and pro-tumour immunity status[20, 22-24], which facilitates the migration, invasion and metastasis of tumour cells[31-35] and affects patients' prognosis with multiple solid tumours[21], including lung[36], liver[37], gastric[38] and colorectal cancer[22-24]. As a component of peripheral blood in patients with cancer, CTC was originally considered to be an important mediator of tumour recurrence and metastasis[6] and was also reported to be significantly associated with poor prognosis in a variety of malignancies[9, 11, 39]. Noteworthily, recent studies have also shown that the release and survival of CTCs is the result of interactions with the host's immune system, and the number of CTCs in the peripheral blood can also indirectly reflect the immune-nutritional status of patients[16-18]. In view of the above findings, we attempted to create a new scoring system by combining both CONUT and CTC (CONUT-CTC), which is theoretically more convincing and reflects a stronger prognostic value for predicting survival. This hypothesis was confirmed in our present study, in which 160 CRC patients treated with curative resection were enrolled. Our results indicated that a positive correlation existed between the preoperative CONUT score and CTC count, and preoperative CONUT-CTC score can serve as an independent biomarker for prognostic estimates. Furthermore, according to ROC curve analysis, the AUC value of the CONUT-CTC score exceeded those of CONUT and $\mathrm{CTC}$, providing a more convincing conclusion that preoperative CONUT-CTC score exhibits comparable prognostic ability that is even stronger than preoperative CONUT score or CTC alone.

With regard to the mechanisms underlying the relationship between CONUT score and CTC, they are multifaceted and remain unclear. A serials of in vitro experiments and clinical studies revealed that the potential mechanisms might be clarified by exploring the function of the aforementioned three components and their effects on the biological behaviour of tumour cells, including proliferation, migration, invasion and survival[32-35, 40-42]. Among these, serum albumin is not only a strong indicator of host nutritional conditions but also relates to the inflammation and immune status of host[43]. Related studies have shown that low serum albumin levels may create favourable conditions for the migration, invasion and colonization of tumour cells by deteriorating the nutritional status and immune status of patients[31]. Lymphocytes have a key role in tumour defence by inducing cytotoxic cell death, and they play an important role in cancer immune surveillance[32]. Low lymphocyte counts weaken the body's immune defence function by initiating a cytotoxic immune response, thereby exerting a pro-tumour effect and enhancing the proliferation, growth and invasion of tumour cells[40, 41]. Among the three above components, total cholesterol level is the main difference between the CONUT score and other inflammatory markers. As an important component of the cell
Figure 6. Time-dependent ROC curves of preoperative CONUT score, CTC status, CONUT-CTC score, and PTNM stage for the prediction of CRC patients' outcomes. A. Recurrence-free survival. B. Cancer-specific survival. Abbreviations: CRC, colorectal cancer; CONUT, controlling nutritional status; CTC, circulating tumour cell; ROC, receiver operating characteristic; AUC, area under curve; $\mathrm{Cl}$, confidence interval. 
membrane, cholesterol participates in a variety of signalling pathways related to tumourigenesis, progression, and immune response[34, 35]. Previous studies have confirmed that lower serum total cholesterol levels are associated with poor prognosis in CRC[42], which may contribute to tumour progression by impairing the immune system, affecting signalling in tumour cells, and promoting tumour cell proliferation, migration, and invasion[33, 34]. Based on these findings, we deduced that the high CONUT score reflects the poor nutrition and low anti-tumour immune status of host, which creates favourable conditions for the proliferation, migration and invasion of tumour cells in tumour lesions, thereby promoting the release of CTCs. Meanwhile, the decrease of lymphocyte counts in the peripheral blood makes the CTCs less likely to be attacked by immune cells after entering the bloodstream, so that more CTCs survive and are detected. This also provided a reasonable explanation for the findings of our study on the relationship between high preoperative CONUT-CTC score and multiple unfavourable clinicopathological features, including depth of tumour invasion, pTNM stage, lymphatic vessel invasion and venous invasion, which were consistent with previous studies[9, 11, 22-24].

In the past few decades, many clinicopathological biomarkers have been identified to efficiently predict recurrence and survival in CRC through the continuous efforts of researchers[44]. However, expensive and technical limitations have prevented their clinical spread. Blood-based biomarkers are now recognized to be attractive practical tools due to the advantages of being easily accessible, routinely tested, relatively noninvasive and inexpensive[45]. Additionally, CTCBIOPSY ${ }^{\circledR}$ (Wuhan YZY Medical Science and Technology Co., Ltd., Wuhan, China), the device used in our study for CTC isolation, was jointly developed by us and Medical Science and Technology Co., Ltd.[16]. As a device based on the principle of isolation by size of epithelial tumour cells (ISET), it has been demonstrated to achieve CTC separation in a manner of cheapness, automation and efficiency. Therefore, CONUT-CTC score, as a blood-derived tool, has the advantages of being easily measurable, cost-efficient and highly repeatable in clinical practice. Combined with our results, preoperative CONUT-CTC score may be a novel and valuable tool for custom surveillance schedules and individualized therapeutic strategies for CRC patients of different risk subgroups. For CRC patients with a high preoperative CONUT-CTC score, more intense therapy and more frequent follow-up after curative resection might be needed.

There were several limitations in this study.
First, the study was a retrospective study from a single centre. Second, the sample size was relatively small, which made it difficult to conduct further verification analysis by dividing patients into training and validation cohorts. Third, the follow-up time was relatively short, and not all patients received standardized adjuvant therapy and follow-up procedures according to the NCCN guidelines, therefore, our results should be interpreted with caution and require more detailed and accurate data to further verify. In addition, we did not routinely measure and analyse other blood-derived immunenutritional markers, such as PNI or Glasgow prognostic score (GPS). Nevertheless, there are no studies focusing on the relationships between preoperative CONUT score and CTC in CRC patients treated with curative resection. These preliminary findings can lay the foundation for future larger, longer and more standardized follow-up study on this topic, which are required to confirm our results and to assess the validity of this prognostic indicator.

In conclusion, our study provides the first evidence that preoperative CONUT-CTC score is associated with tumour progression and can be considered an independent prognostic factor for predicting RFS and CSS in CRC patients treated with curative resection. As a novel, economical and reliable biomarker, it may have great clinical potential for individualized treatment decision-making and improving follow-up performance.

\section{Supplementary Material}

Supplementary figures and tables.

http://www.ijbs.com/v15p1325s1.pdf

\section{Acknowledgements}

This work was supported in part by the following funds: (1) Science Fund of the National Natural Science Foundation of China (grant numbers: 81572874 and 81702411); (2) Zhongnan Hospital of Wuhan University, Technology and Innovation Seed Found (grant number: znpy2016058). The authors also appreciate Wuhan YZY Medical Science and Technology Co., Ltd. for providing equipment and excellent technical support in CTC detection.

\section{Competing Interests}

The authors have declared that no competing interest exists.

\section{References}

1. Bray F, Ferlay J, Soerjomataram I, Siegel RL, Torre LA, Jemal A. Global cancer statistics 2018: GLOBOCAN estimates of incidence and mortality worldwide for 36 cancers in 185 countries. CA Cancer J Clin. 2018; 68: 394-424.

2. Miller KD, Siegel RL, Lin CC, Mariotto AB, Kramer JL, Rowland JH, et al. Cancer treatment and survivorship statistics, 2016. CA Cancer J Clin. 2016; 66: 271-89. 
3. Lambert AW, Pattabiraman DR, Weinberg RA. Emerging Biological Principles of Metastasis. Cell. 2017; 168: 670-91.

4. Schirripa M, Lenz HJ. Biomarker in Colorectal Cancer. Cancer J. 2016; 22: 156-64

5. Plaks V, Koopman CD, Werb Z. Cancer. Circulating tumor cells. Science. 2013; 341: 1186-8.

6. Massague J, Obenauf AC. Metastatic colonization by circulating tumour cells. Nature. 2016; 529: 298-306.

7. Micalizzi DS, Maheswaran S, Haber DA. A conduit to metastasis: circulating tumor cell biology. Genes Dev. 2017; 31: 1827-40.

8. Pantel K, Alix-Panabieres C. Liquid biopsy in 2016: Circulating tumour cells and cell-free DNA in gastrointestinal cancer. Nat Rev Gastroenterol Hepatol. 2017; 14: 73-4.

9. Yang C, Zou K, Zheng L, Xiong B. Prognostic and clinicopathological significance of circulating tumor cells detected by RT-PCR in non-metastatic colorectal cancer: a meta-analysis and systematic review. BMC Cancer. 2017; 17: 725 .

10. Ma B, King AD, Leung L, Wang K, Poon A, Ho WM, et al. Identifying an early indicator of drug efficacy in patients with metastatic colorectal cancer-a prospective evaluation of circulating tumor cells, 18F-fluorodeoxyglucose positron-emission tomography and the RECIST criteria. Ann Oncol. 2017; 28: 1576-81.

11. Yang C, Shi D, Wang S, Wei C, Zhang C, Xiong B. Prognostic value of pre- and post-operative circulating tumor cells detection in colorectal cancer patients treated with curative resection: a prospective cohort study based on ISET device. Cancer Manag Res. 2018; 10: 4135-44.

12. Tan K, Leong SM, Kee Z, Caramat PV, Teo J, Blanco MVM, et al. Longitudinal monitoring reveals dynamic changes in circulating tumor cells (CTCs) and CTC-associated miRNAs in response to chemotherapy in metastatic colorectal cancer patients. Cancer Lett. 2018; 423: 1-8.

13. Zieglschmid V, Hollmann C, Bocher O. Detection of disseminated tumor cells in peripheral blood. Crit Rev Clin Lab Sci. 2005; 42: 155-96.

14. Chang YS, di Tomaso E, McDonald DM, Jones R, Jain RK, Munn LL. Mosaic blood vessels in tumors: frequency of cancer cells in contact with flowing blood. Proc Natl Acad Sci U S A. 2000; 97: 14608-13.

15. Grivennikov SI, Greten FR, Karin M. Immunity, inflammation, and cancer. Cell. 2010; 140: 883-99.

16. Chen F, Wang S, Fang Y, Zheng L, Zhi X, Cheng B, et al. Feasibility of a novel one-stop ISET device to capture CTCs and its clinical application. Oncotarget. 2017; 8: 3029-41.

17. Yang C, Zhang N, Wang S, Shi D, Zhang C, Liu K, et al. Wedge-shaped microfluidic chip for circulating tumor cells isolation and its clinical significance in gastric cancer. J Transl Med. 2018; 16: 139.

18. Zheng L, Zou K, Yang C, Chen F, Guo T, Xiong B. Inflammation-based indexes and clinicopathologic features are strong predictive values of preoperative circulating tumor cell detection in gastric cancer patients. Clin Transl Oncol. 2017; 19: 1125-32.

19. Onodera T, Goseki N, Kosaki G. [Prognostic nutritional index in gastrointestinal surgery of malnourished cancer patients]. Nihon Geka Gakkai Zasshi. 1984; 85: 1001-5.

20. Ignacio de Ulibarri J, Gonzalez-Madrono A, de Villar NG, Gonzalez P, Gonzalez B, Mancha A, et al. CONUT: a tool for controlling nutritional status. First validation in a hospital population. Nutr Hosp. 2005; 20: 38-45.

21. Liang RF, Li JH, Li M, Yang Y, Liu YH. The prognostic role of controlling nutritional status scores in patients with solid tumors. Clin Chim Acta. 2017; 474: $155-8$.

22. Iseki $Y$, Shibutani M, Maeda K, Nagahara H, Ohtani H, Sugano K, et al. Impact of the Preoperative Controlling Nutritional Status (CONUT) Score on the Survival after Curative Surgery for Colorectal Cancer. PLoS One. 2015; 10: e0132488.

23. Tokunaga R, Sakamoto Y, Nakagawa S, Ohuchi M, Izumi D, Kosumi K, et al. CONUT: a novel independent predictive score for colorectal cancer patients undergoing potentially curative resection. Int J Colorectal Dis. 2017; 32: 99-106.

24. Daitoku N, Miyamoto Y, Tokunaga R, Sakamoto Y, Hiyoshi Y, Iwatsuki M, et al. Controlling Nutritional Status (CONUT) Score Is a Prognostic Marker in Metastatic Colorectal Cancer Patients Receiving First-line Chemotherapy. Anticancer Res. 2018; 38: 4883-8.

25. Edge SB, Compton CC. The American Joint Committee on Cancer: the 7th edition of the AJCC cancer staging manual and the future of TNM. Ann Surg Oncol. 2010; 17: 1471-4.

26. Clavien PA, Barkun J, de Oliveira ML, Vauthey JN, Dindo D, Schulick RD, et al. The Clavien-Dindo classification of surgical complications: five-year experience. Ann Surg. 2009; 250: 187-96.

27. Benson AB, 3rd, Venook AP, Bekaii-Saab T, Chan E, Chen YJ, Cooper HS, et al. Rectal Cancer, Version 2.2015. J Natl Compr Canc Netw. 2015; 13: 719-28; quiz

28. Benson $\mathrm{AB}, 3 \mathrm{rd}$, Venook AP, Cederquist L, Chan E, Chen $\mathrm{YJ}$, Cooper HS, et al. Colon Cancer, Version 1.2017, NCCN Clinical Practice Guidelines in Oncology. J Natl Compr Canc Netw. 2017; 15: 370-98.

29. Glynne-Jones R, Wyrwicz L, Tiret E, Brown G, Rodel C, Cervantes A, et al. Rectal cancer: ESMO Clinical Practice Guidelines for diagnosis, treatment and follow-up. Ann Oncol. 2017; 28: iv22-iv40.

30. Amin MB, Greene FL, Edge SB, Compton CC, Gershenwald JE, Brookland RK, et al. The Eighth Edition AJCC Cancer Staging Manual: Continuing to build a bridge from a population-based to a more "personalized" approach to cancer staging. CA Cancer J Clin. 2017; 67: 93-9.

31. Liu J, Dai Y, Zhou F, Long Z, Li Y, Liu B, et al. The prognostic role of preoperative serum albumin/globulin ratio in patients with bladder urothelial carcinoma undergoing radical cystectomy. Urol Oncol. 2016; 34: 484 e1- e8.

32. Heinzel S, Marchingo JM, Horton MB, Hodgkin PD. The regulation of lymphocyte activation and proliferation. Curr Opin Immunol. 2018; 51: 32-8.

33. Haghikia A, Landmesser U. High-Density Lipoproteins: Effects on Vascular Function and Role in the Immune Response. Cardiol Clin. 2018; 36: 317-27.

34. Jacobs RJ, Voorneveld PW, Kodach LL, Hardwick JC. Cholesterol metabolism and colorectal cancers. Curr Opin Pharmacol. 2012; 12: 690-5.

35. Wang C, Li P, Xuan J, Zhu C, Liu J, Shan L, et al. Cholesterol Enhances Colorectal Cancer Progression via ROS Elevation and MAPK Signaling Pathway Activation. Cell Physiol Biochem. 2017; 42: 729-42.

36. Shoji F, Haratake N, Akamine T, Takamori S, Katsura M, Takada K, et al. The Preoperative Controlling Nutritional Status Score Predicts Survival After Curative Surgery in Patients with Pathological Stage I Non-small Cell Lung Cancer. Anticancer Res. 2017; 37: 741-7.

37. Harimoto N, Yoshizumi T, Inokuchi S, Itoh S, Adachi E, Ikeda Y, et al. Prognostic Significance of Preoperative Controlling Nutritional Status (CONUT) Score in Patients Undergoing Hepatic Resection for Hepatocellular Carcinoma: A Multi-institutional Study. Ann Surg Oncol. 2018; 25: 3316-23.

38. Liu X, Zhang D, Lin E, Chen Y, Li W, Chen Y, et al. Preoperative controlling nutritional status (CONUT) score as a predictor of long-term outcome after curative resection followed by adjuvant chemotherapy in stage II-III gastric Cancer. BMC Cancer. 2018; 18: 699.

39. Yang C, Zou K, Yuan Z, Guo T, Xiong B. Prognostic value of circulating tumor cells detected with the CellSearch System in patients with gastric cancer: evidence from a meta-analysis. Onco Targets Ther. 2018; 11: 1013-23.

40. Murata M. Inflammation and cancer. Environ Health Prev Med. 2018; $23: 50$.

41. Saito $\mathrm{H}$, Kono $\mathrm{Y}$, Murakami $\mathrm{Y}$, Shishido $\mathrm{Y}$, Kuroda $\mathrm{H}$, Yamamoto $\mathrm{M}$, et al. Prognostic Significance of Pre- and Postoperative Lymphocyte Counts in Patients with Gastric Cancer. Dig Surg. 2018.

42. Li JR, Zhang Y, Zheng JL. Decreased pretreatment serum cholesterol level is related with poor prognosis in resectable non-small cell lung cancer. Int J Clin Exp Pathol. 2015; 8: 11877-83.

43. Rothschild MA, Oratz M, Schreiber SS. Serum albumin. Hepatology. 1988; 8: 385-401.

44. Erstad DJ, Tumusiime G, Cusack JC, Jr. Prognostic and Predictive Biomarkers in Colorectal Cancer: Implications for the Clinical Surgeon. Ann Surg Oncol. 2015; 22: 3433-50.

45. Yoruker EE, Holdenrieder S, Gezer U. Blood-based biomarkers for diagnosis, prognosis and treatment of colorectal cancer. Clin Chim Acta. 2016; 455: 26-32. 